\title{
Um componente de network slicing para o suporte de multi-inquilinos nas RANs do LTE.
}

\author{
Pedro H. A. Rezende ${ }^{1}$ e Edmundo R. M. Madeira ${ }^{1}$ \\ ${ }^{1}$ Instituto da Computação \\ Universidade Estadual de Campinas (Unicamp) \\ Campinas, São Paulo, Brasil
}

pedrohenriquedIrc.ic.unicamp.br, edmundodic.unicamp.br

\begin{abstract}
G networks intend to integrate network slicing into their architecture aiming to satisfy the different service levels of an abundant amount of devices. Network Slicing relies on softwarization technologies, such as SDN and NFV, to instantiate slices (virtual networks) on top of the same physical substrate. This work introduces the "Otimizador de Slices", a component developed as an extension of LTE's evolved NodeB, responsible to perform network slicing for LTE downlink transmission. This component receives slice's information from multiple Service Providers and, based on the analysis of these information and on the network state, the proposed component selects the best slice to be scheduled at the moment. Simulations were performed to validate our proposal and expose the benefits that can be obtained by it, such as an enhancement of end user's QoS experience.
\end{abstract}

Resumo. As redes $5 G$ pretendem integrar "network slicing" em sua arquitetura visando satisfazer os diferentes níveis de serviços de uma quantidade massiva de dispositivos. Network Slicing apoia-se em tecnologias de softwarização, como SDN e NFV, para instanciar slices (redes virtuais) sobre um mesmo substrato físico. Esses slices são mutualmente independentes e customizados de acordo com as necessidades dos usuários. Este trabalho introduz o Otimizador de Slices, um componente desenvolvido como uma extensão do evolved NodeB das redes LTE, responsável por concretizar o conceito de network slicing na transmissão de tráfego downlink das redes LTE. Este componente recebe informações sobre slices oriundas de múltiplos Provedores de Serviços e, a partir da análise dessas informações e do estado da rede, o componente proposto seleciona o melhor slice a ser escalonado no momento. Simulações foram realizadas para avaliar nossa proposta e mostrar os benefícios dela, como a melhora da QoS ofertada aos usuários finais.

\section{Introdução}

As futuras redes $5 \mathrm{G}$ pretendem satisfazer os diferentes requisitos de serviço de uma quantidade abundante de aplicações. Para isso, os sistemas $5 \mathrm{G}$ planejam aumentar os recursos oferecidos aos usuários por meio de novas tecnologias de acesso de rádio e bandas no espectro, tais como MIMO (múltiplas entradas, múltiplas saídas) massivo e ondas milimétricas. Ademais, as redes $5 \mathrm{G}$ não empregarão a arquitetura monolítica presente nas redes atuais, uma vez que tal arquitetura é incapaz de atender os diferentes requisitos de serviço dos mais diversos casos de uso. Tais casos de uso incluem, por exemplo, veículos autônomos, hospitais inteligentes e tecnologias vestíveis. 
Para suportar os mais diversos casos de uso, as redes $5 \mathrm{G}$ visam integrar o conceito de Network Slicing [Foukas et al. 2017] em sua arquitetura. Embora Network Slicing não seja uma tecnologia nova, apenas recentemente ela foi introduzida nas redes sem fio. Network Slicing permite que operadores de rede criem múltiplas redes virtuais (ou slices) sobre uma mesma infraestrutura física. Esses slices são mutualmente independentes e customizados de acordo com os requisitos definidos entre os operadores de rede e os usuários finais. A virtualização da infraestrutura de rede pode ser realizada, por exemplo, a partir de um hypervisor [Blenk et al. 2016].

O conceito de Network Slicing em redes $5 \mathrm{G}$ é realizado graças à consolidação de tecnologias de softwarização de redes, como Redes Definidas por Software (Software Defined Networks - SDN) [Kreutz et al. 2015] e Virtualização das Funções de Rede (Network Functions Virtualization - NFV) [Mijumbi et al. 2016]. Essas tecnologias de softwarização visam trazer os benefícios do software às redes. A flexibilidade, programabilidade e modularidade são exemplos de tais benefícios.

Network Slicing pode ser realizado tanto nas redes de acesso (Radio Access Networks - RAN) quanto no núcleo dos sistemas 5G. Recursos de computação, armazenamento, espectro, bem como as funções de rede podem ser virtualizadas e distribuídas aos diferentes slices pertencentes à rede. Neste trabalho, o foco é a realização do conceito de Network Slicing nas redes de acesso dos sistemas 5G, mais especificamente na alocação de espectro do canal downlink da RAN. Para isso, propomos um componente, chamado de Otimizador de Slices (OS), desenvolvido como uma extensão do evolved NodeB (eNB) das redes Long Term Evolution (LTE). As redes LTE são usadas como base para as futuras redes $5 \mathrm{G}$.

O OS permite que múltiplos Provedores de Serviço (Service Providers - SPs) criem slices. O OS recebe mensagens sobre slices por intermédio de um hypervisor. Os múltiplos SPs oferecem seus serviços aos usuários finais por meio de controladores SDN, os quais operam em cima do hypervisor. O OS, além de concretizar o conceito de network slicing, é responsável por adaptar os slices ao estado da rede. Nossa proposta foi implementada e avaliada no NS-3 [NS-3 2017]. Os resultados obtidos mostram a eficiência de nossa proposta, como a melhora da Qualidade de Serviço (Quality of Service - QoS) percebida pelos usuários finais.

As demais seções deste trabalho estão organizadas da seguinte forma. A Seção 2 apresenta alguns conceitos importantes para o restante do trabalho, enquanto a Seção 3 introduz alguns trabalhos sobre network slicing presentes na literatura. A Seção 4 apresenta a nossa arquitetura do sistema e o componente proposto, bem como suas implementações. A Seção 5 expõe a avaliação de desempenho de nossa proposta. A Seção 6 conclui o artigo e apresenta trabalhos futuros.

\section{Fundamentação Teórica}

Nós apresentaremos nesta seção uma visão geral sobre Redes Sem Fio Definidas por Software, transmissão de tráfego downlink em redes LTE e rede multi-inquilinos.

\subsection{Redes Sem Fio Definidas por Software}

SDN separa o plano de controle do plano de dados dos dispositivos de encaminhamento de pacotes. O plano de controle, que torna-se uma entidade externa, é consolidado em componentes de software, chamado de controlador, o que facilita a gerência de redes. 
Esse controlador é responsável pela programação de dispositivos por meio da Application Programming Interface (API) Southbound e pelo provisionamento de serviços para aplicações a partir da API Northbound. Os dispositivos de rede pertencentes ao plano de dados tornam-se simplificados, realizando um pequeno número de tarefas, dentre elas, o encaminhamento de pacotes.

Grande parte dos esforços em SDN tem sido feito nas redes cabeadas; entretanto, uma arquitetura de redes sem fio baseada em SDN pode oferecer inúmeros benefícios tanto aos operadores de rede quanto aos usuários finais [Haque and Abu-Ghazaleh 2016]. Em redes celulares, uma arquitetura baseada em SDN pode, por exemplo, facilitar a alocação de recursos de rádio, auxiliar no processo de handover, distribuir informações relacionadas ao estado das células e ajudar na redução de interferência entre elas.

\subsection{Transmissão de tráfego downlink em redes LTE}

O escalonador downlink na camada MAC do eNB é responsável pelo escalonamento de pacotes downlink [Grøndalen et al. 2017]. O Physical Downlink Shared Channel (PDSCH) é empregado para a transmissão de dados downlink do eNB aos equipamentos dos usuários (User Equipments - UEs). Todas as transmissões estão organizadas em quadros de rádio de $10 \mathrm{~ms}$ cada. Em cada quadro há 10 subquadros de $1 \mathrm{~ms}$ cada, que é o Intervalo de Tempo de Transmissão (Transmission Time Interval - TTI).

Em cada TTI, o escalonador realiza a alocação de recursos de rádio. Essa alocação é feita a partir de algumas informações, tais como: o estado do buffer da entidade Radio Link Control (RLC); a QoS recebida de camadas superiores; e do Indicador de Qualidade de Canal (Channel Quality Indicator - CQI), que é reportado pelos UEs. As restrições de QoS são fornecidas pelos bearers do Evolved Packet System (EPS). Tais bearers são associados a um Identificador de Classe de QoS (QoS Class Identifier - QCI) que, por sua vez, define alguns parâmetros, como a prioridade de escalonamento e perda de pacotes aceitável. O esquema Adaptive Modulation and Coding (AMC) seleciona uma modulação e codificação adequada para a transmissão de pacotes com o intuito de aumentar a eficiência da rede. A camada MAC também possui a entidade Hybrid Automatic Repeat Request (HARQ) para a retransmissão de pacotes perdidos.

\subsection{Rede multi-inquilinos}

As operadoras móveis vêm tendo dificuldade de acomodar em sua infraestrutura de rede o tráfego de seus usuários, uma vez que houve um aumento expressivo desse tráfego nos últimos anos devido à popularização dos smartphones e tablets, bem como do vídeo sob demanda. Dessa forma, as operadoras móveis têm um alto gasto operacional e de infraestrutura para acomodar esse tráfego crescente. Com isso, muitas operadoras móveis estão compartilhando entre si suas infraestruturas de rede. O compartilhamento de uma infraestrutura de rede é comumente chamado de rede multi-inquilinos [Samdanis et al. 2016]. O principal benefício de redes multi-inquilinos é a redução dos custos de capital e operacional para todos os envolvidos nesse compartilhamento.

\section{Trabalhos Relacionados}

Os autores em [Caballero et al. 2017] propõem um framework baseado em um modelo de alocação proporcional de recursos com o objetivo de realizar o conceito de network slicing em redes multi-inquilinos. O framework proposto permite o compartilhamento dinâmico de recursos entre os slices, aumentando o desempenho geral dos inquilinos. Além disso, 
tal framework é genérico e não tem como base uma tecnologia celular específica. Em [Kokku et al. 2013], os autores introduzem CellSlice, um sistema que realiza o conceito de network slicing em redes de acesso na tecnologia WiMAX. Diferente de nossa proposta, ambos os trabalhos não focam nas redes LTE e não usam uma arquitetura baseada em SDN.

Em [Chartsias et al. 2017] os autores propõem um solução baseada em SDN para a gerência e orquestração de slices de múltiplos inquilinos. Todavia, diferente de nosso trabalho, em [Chartsias et al. 2017] a rede de acesso é baseada na tecnologia WiFi e o foco de [Chartsias et al. 2017] é no provisionamento de slices no backhaul das redes sem fio. É proposto em [Choyi et al. 2016] um framework que, a partir dos descritores de serviços definidos, realiza a negociação, seleção e atribuição dos slices na rede 5G aos respectivos usuários. Entretanto, em [Choyi et al. 2016] não são realizados experimentos para validar o framework proposto.

Em [Hu et al. 2016], os autores apresentam um mecanismo de escalonamento e slicing dinâmico para as redes LTE. No mecanismo proposto, uma quantidade de subcanais é alocada para cada slice. Tais sub-canais podem ser realocados entre os slices com o objetivo de garantir os diferentes acordos de nível de serviço. $\mathrm{O}$ trabalho proposto em [Kamel et al. 2014] expõe um sistema para fatiar uma rede LTE em múltiplas redes virtuais. Após o fatiamento, tais redes virtuais são atribuídas a diferentes Provedores de Serviços e, baseado em um contrato de serviço, uma quantidade mínima de blocos de recurso é alocada a cada Provedor de Serviço.

Os autores em [Parsaeefard et al. 2015] apresentam um mecanismo de provisionamento de recursos e controle de admissão com o objetivo de maximizar o desempenho dos slices a partir da informação do estado do canal dos dispositivos dos usuários. A principal diferença entre nosso trabalho e [Hu et al. 2016, Kamel et al. 2014, Parsaeefard et al. 2015] é que nossa proposta usa uma arquitetura baseada em SDN. Em um trabalho anterior [Rezende and Madeira 2018], nós realizamos o conceito de network slicing na transmissão de tráfego downlink das redes LTE. Neste trabalho nós estendemos [Rezende and Madeira 2018], apresentando e implementando uma arquitetura multiinquilinos. Dessa forma, permitimos que múltiplos SPs atuem na rede.

\section{Arquitetura do Sistema e Componente proposto}

Nesta seção serão apresentados a nossa arquitetura do sistema e o Otimizador de Slices, desenvolvido como uma extensão do eNB das redes LTE.

\subsection{Visão Geral}

A Figura 1 ilustra nossa arquitetura do sistema, que é constituída pelo Provedor de Infraestrutura (PI), o qual é proprietário de toda a infraestrutura física de rede; pelos controladores vSDNs e pelo hypervisor; além de toda infraestrutura física LTE, tanto da RAN quanto do núcleo (Evolved Packet Core - EPC). O PI é responsável por alocar recursos de rede aos Provedores de Serviços e pela gerência de tais recursos. Para a alocação desses recursos, o PI baseia-se nos Acordos de Níveis de Serviços (Service Level Agreements SLAs) estabelecidos com os SPs.

A entidade Funções de Gerência e Orquestração (Management and Orchestration - MANO) presente no PI é responsável por alocar os recursos de rede aos SPs a partir dos SLAs definidos entre o PI e os SPs. Para isso, a MANO alimenta periodicamente 


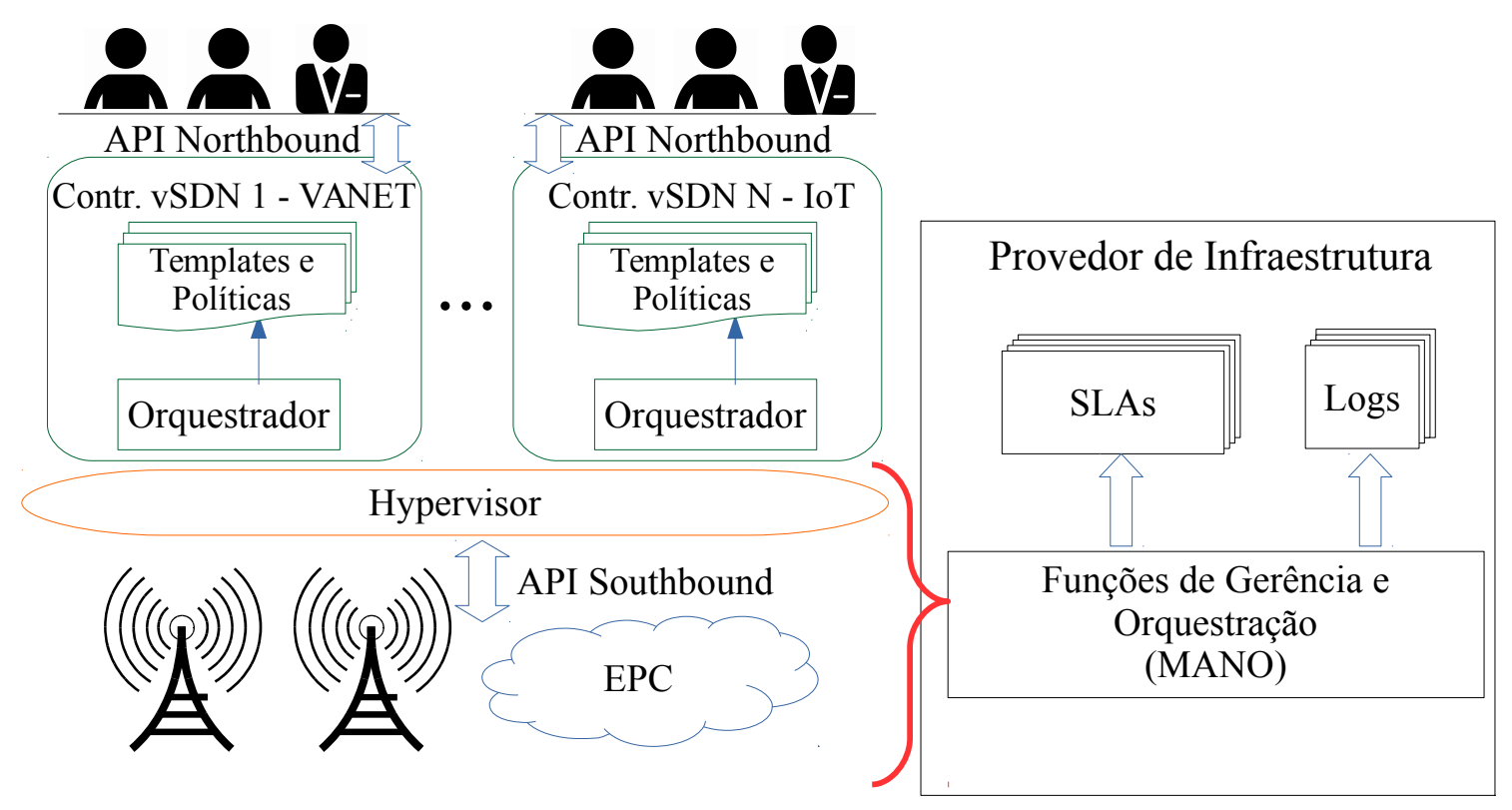

Figura 1. Visão Geral da Arquitetura do Sistema.

um hypervisor (Ex.: OpenVirteX [Al-Shabibi et al. 2014]) com informações relativas aos recursos contratados em cada SP. A quantidade de recursos de rádio ou o número de eNBs contratados são exemplos dessas informações. Uma vez que um quadro LTE contém 10 subquadros, os recursos de rádio são alocados pelo PI em um vetor de dez posições, chamado de Vetor de Mapeamento (VDM), onde cada posição representa a numeração do subquadro e o valor corresponde ao identificador do SP. É criado um VDM para cada eNB no sistema. O hypervisor, após receber o VDM, envia-o ao OS, que usará esse vetor para saber qual SP tem a prioridade de escalonamento neste subquadro. Além disso, o hypervisor, a partir dessas informações recebidas pelo PI, abstrai os recursos físicos de rede, alocando-os aos SPs como virtual Software Defined Networks (vSDNs). Cada SP, por sua vez, utiliza o seu próprio sistema operacional de rede (controlador) para configurar a sua vSDN.

Ao receber a vSDN, o controlador (e.g. POX ${ }^{1}$, Floodlight ${ }^{2}$ ) é responsável por alocar os recursos virtualizados aos seus usuários. Para isso, cada SP define seus templates e políticas de orquestração de recursos em seu controlador. Templates podem ser definidos, por exemplo, para tráfego de vídeo e tráfego de voz, onde cada um desses tráfegos tem restrições distintas de QoS. Esses templates são acessados pelos usuários finais a partir da API Northbound, que é disponibilizada pelo controlador. Por meio dessa mesma API, os operadores de rede definem suas políticas de alocação de recursos. Em todos os controladores há um orquestrador que recebe como entrada: os recursos de rede, as políticas e os templates, e gera como saída dois parâmetros: os descritores de slices (DSs) e um vetor de alocação (VA). Os DSs contêm informações acerca dos slices, por exemplo, o conjunto de tuplas contendo os fluxos registrados no slice, assim como o identificador e a prioridade de cada slice. Nesse conjunto de tuplas, cada tupla é composta por dois itens, o International Mobile Subscriber Identity (IMSI) e o QCI. São necessários esses dois parâmetros uma vez que um mesmo UE pode gerar tráfegos pertencentes a diferentes

\footnotetext{
${ }^{1}$ https://github.com/noxrepo/pox

${ }^{2}$ http://www.projectfloodlight.org/floodlight/
} 
slices. O VA define a distribuição dos recursos de rádio aos slices.

Os DSs e o VA gerados pelo orquestrador são enviados ao hypervisor. Ao receber ambos os parâmetros, o hypervisor verifica a integridade deles e, caso haja algum problema com eles, o hypervisor envia uma mensagem ao MANO que, por sua vez, escreve os erros em um arquivo de $\log$. Se os DSs e o VA estiverem corretos, uma coleção de pares chave-valor é criada para mapear os valores do VA em subquadros de rádio. Tal coleção é chamada de mapa de alocação (MA). O MA e os DSs são enviados pelo hypervisor ao OS por meio da API Southbound. Exemplos de protocolos que podem ser usados na API Southbound incluem o OpenFlow [McKeown et al. 2008] e o NETCONF.

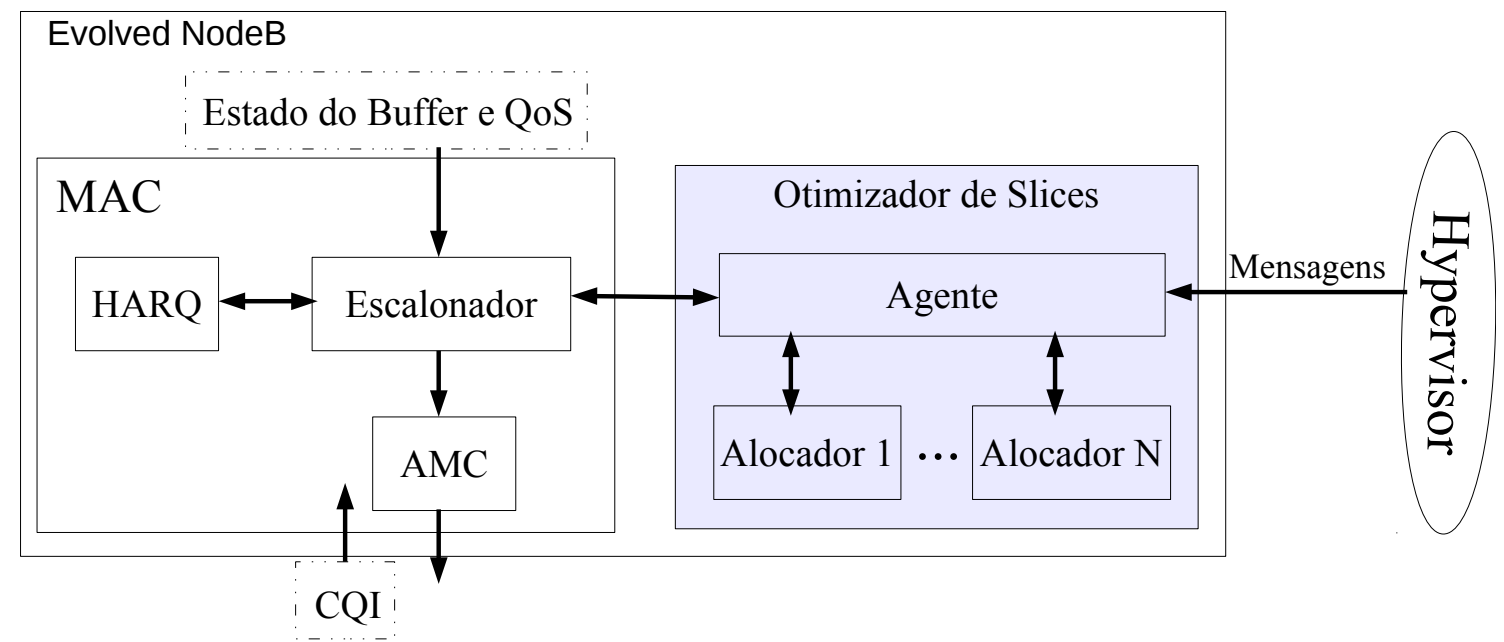

Figura 2. Otimizador de Slices e entidades relevantes para a transmissão de tráfego downlink.

A Figura 2 ilustra o Otimizador de Slices, representado pela cor azul, assim como as entidades da camada MAC relevantes para a transmissão de dados downlink. O OS precisa ser inserido em cada um dos eNBs na rede para que eles possam comunicar com o hypervisor. O OS possui um módulo Agente e vários Alocadores, onde a quantidade de Alocadores é a mesma do número de SPs atuando na rede. O hypervisor, além de entregar os DSs e o MA de cada SP ao Agente, também envia uma requisição para a criação de um novo Alocador sempre que um SP é adicionado à rede. Esse novo Alocador estará vinculado ao SP e, portanto, sempre que o Agente receber os DSs e o MA de um SP, ambos os parâmetros serão entregues ao Alocador associado ao respectivo SP.

O Agente recebe do Escalonador dois parâmetros: o subquadro de rádio atual e um conjunto contendo o estado do buffer de cada fluxo na rede. A partir desses dois parâmetros, o Agente define o SP responsável por transmitir nesse subquadro e, subsequentemente, envia uma permissão de transmissão e o subquadro atual de rádio ao Alocador que corresponde ao SP escolhido. O Alocador ao receber essa permissão e o subquadro escolhe o slice a ser escalonado no momento. Essa escolha é feita tendo como base os DSs e o MA do respectivo SP. Após a escolha do slice, o Alocador envia ao Agente um conjunto de tuplas contendo os fluxos que pertencem ao slice escolhido. $\mathrm{O}$ Agente, ao receber esse conjunto, envia-o ao Escalonador para que o mesmo aloque os blocos de recurso aos UEs do conjunto. 


\subsection{Detalhes de Implementação}

A implementação da arquitetura e do OS foi realizada no NS-3. O NS-3 é um simulador de rede de eventos discretos desenvolvido em C++. O NS-3 implementa as redes LTE no módulo LENA [LENA 2017]. A nossa implementação é basicamente composta por seis classes C++. São elas: OS, Alocador, hypervisor, PI, controlador vSDN e orquestrador. O NS-3 não fornece um protocolo para a comunicação do eNB com uma entidade externa à rede LTE. Dessa forma, a comunicação do OS com o hypervisor, bem como entre o hypervisor e os controladores vSDNs, é feita por meio de chamadas de função $\mathrm{C}++$. Similarmente, a API Northbound exposta pelos controladores vSDNs e a comunicação entre o hypervisor e o PI são chamadas de funções C++. Em nossa implementação, um slice é um conjunto de fluxos pertencendo ao mesmo tipo de aplicação. Um slice contém fluxos de apenas um único SP, não podendo ser compartilhado com outro SP. Foi implementada a comunicação entre o hypervisor e um único eNB. Além disso, não há comunicação entre o hypervisor e o EPC, visto que o foco deste trabalho é no provisionamento de slices na rede de acesso.

O PI é responsável por gerenciar a infraestrutura de rede. Atualmente, a única função implementada no PI é o provisionamento de recursos definidos nos SLAs. Apenas os recursos de rádio no canal downlink são ofertados aos SPs. Ou seja, no SLA de cada SP é definido o identificador e uma quantidade mínima de subquadros que podem ser disponibilizados aos SPs. Dessa forma, os 10 subquadros de rádio são divididos entre os SPs a partir da análise dos SLAs, e armazenados no VDM. Em nossa implementação, definimos dois SLAs. Um deles para um SP que proverá conexão de rede a veículos e um outro SLA para um SP oferecendo os recursos de rede às pessoas participando de um evento. Ambos os SPs operam na mesma região. No SLA do SP responsável pelos veículos foram garantidos no mínimo quatro subquadros, enquanto que no outro foram assegurados no mínimo seis subquadros. Com isso, o PI por meio de sua função de provisionamento de recursos (MANO) cria o VDM, que sempre terá dez posições, e distribui aleatoriamente os identificadores dos SPs nele, sempre respeitando o número de subquadros definidos nos SLAs. Uma vez que em nossa implementação o número de subquadros requisitados pelos SPs é o mesmo do total de subquadros de um quadro, ou seja, 10, os SPs receberão o mínimo de subquadros definidos nos SLAs.

Após a criação e o preenchimento do VDM, o PI envia-o ao hypervisor, que por sua vez salva uma cópia desse vetor, e o envia ao OS. Ademais, o hypervisor conta quantas vezes o identificador de cada SP apareceu no VDM, enviando ao controlador vSDN de cada SP sua respectiva soma. A partir dessa soma, o controlador vSDN saberá o número de subquadros disponibilizados a ele. O controlador vSDN não terá acesso aos índices dos subquadros alocados a ele pelo PI, sendo essa uma informação escondida pelo hypervisor. Ao receber o número de subquadros disponibilizados, o orquestrador, que é um objeto instanciado pelo controlador vSDN, cria e aloca os subquadros aos slices baseandose nas políticas definidas pelos operadores de rede e na popularidade dos templates. $\mathrm{O}$ orquestrador gera como saída os DSs e o VA. O VA é um vetor de $N$ posições, onde $N$ é o número de subquadros disponibilizados ao controlador vSDN pelo PI. Nesse VA, os identificadores dos slices são distribuídos de acordo com a alocação computada pelo orquestrador. Dessa forma, se um slice receber três subquadros, então três das $N$ posições disponíveis no VA serão alocadas para esse slice. Tanto os DSs quanto o VA são enviados ao hypervisor pelo controlador vSDN.

Em nossa implementação, o SP responsável pela rede de veículos instancia dois 
slices, um para voz e um outro para vídeo. Cada slice recebe dois subquadros. Já o SP responsável pelas pessoas participando de um evento instancia dois slices, um para tráfego TCP e um outro para vídeo. O primeiro recebe dois subquadros, enquanto que o segundo recebe quatro subquadros. Cada slice possui um DS, que contém os seguintes campos: o identificador do slice, a prioridade do slice e um conjunto contendo os IMSIs de todos os UEs pertencentes ao slice. É possível que um UE esteja em mais de um slice ao mesmo tempo. Nesse caso, o QCI seria usado para distinguir os slices. Entretanto, o NS-3 não suporta completamente o escalonamento por fluxo. Dessa forma, mesmo que diferentes bearers fossem empregados a um mesmo UE, o que representaria slices distintos, apenas um bearer seria usado pelo Escalonador. Portanto, em nossa implementação assumimos que um UE pertence a apenas um slice.

Ao receber os DSs e o VA do controlador vSDN, o hypervisor constrói o conjunto de pares chave-valor, o MA, a partir do VA e o VDM. No MA, a chave é o subquadro e o valor é o identificador do slice. Cada SP possui seu próprio MA. Os DSs e o MA são encapsulados em uma mensagem e enviados ao OS, mais especificamente ao Agente. Nessa mensagem, também há o identificador do SP responsável por gerar os DSs. Após receber essa mensagem, o Agente envia os DSs e o MA ao Alocador vinculado ao controlador vSDN. Em cada TTI, o OS recebe do Escalonador dois parâmetros: o subquadro atual e um vetor contendo o estado do buffer de cada UE. Esse buffer é atualizado pela entidade RLC sempre que dados entram ou saem do buffer RLC.

O Algoritmo 1 mostra o processo de seleção realizado pelo OS para a escolha do slice a ser escalonado. Em cada TTI, o Escalonador downlink requisita ao Agente um slice a ser escalonado. O Agente, por sua vez, chama o procedimento RetornarMelhorSlice, passando como parâmetros o subquadro atual (subQuadro) e o vetor contendo o estado dos buffers de todos os UEs (vetor EB). Nas linhas 2-4, o algoritmo seleciona o SP definido nesse subquadro e verifica se algum UE pertencente a algum dos slices definidos por esse SP tem dados a serem transmitidos (linhas 14-19). O algoritmo vai para a linha 6 se algum UE tiver dados a serem transmitidos; caso contrário, vai para a linha 8 . Na linha 6, o algoritmo seleciona o slice a ser escalonado nesse subquadro e retorna ao Escalonador (linha 7) os UEs que pertencem a esse slice.

O melhor slice é computado pelo procedimento SelecionaSlice (linhas 21-34). Neste procedimento, o slice escolhido é o definido na coleção de pares chave-valor da linha 22 (mapaAlocacao) para esse subquadro. Entretanto, caso nenhum dos UEs pertencentes a esse slice tenha dados a serem transmitidos, o procedimento seleciona o slice com maior prioridade que tenha dados a transferir.

No laço for das linhas 8-12 o algoritmo escolhe o slice do SP de maior prioridade que tenha dados a transmitir. Na linha 12, o algoritmo retorna o conjunto de UEs (IMSIs) pertencentes ao slice para o Escalonador. Após receber o conjunto de IMSIs, o Escalonador converte esse conjunto para uma coleção de Radio Network Temporary Identifiers (RNTIs), pois os escalonadores do NS-3 não trabalham com o IMSI. Posteriormente, o Escalonador aloca os recursos de rádio downlink aos UEs de acordo com sua política.

Conforme pode ser visto no Algoritmo 1, o SP definido pelo PI no subquadro atual sempre tem maior prioridade; entretanto, caso nenhum UE pertencente aos slices instanciados por esse SP tenha dados a serem transmitidos, um outro SP será escolhido para transmitir nesse subquadro. Dessa forma, os recursos de rádio são usados mais eficientemente, uma vez que o Escalonador vai alocar recursos para um slice que realmente 


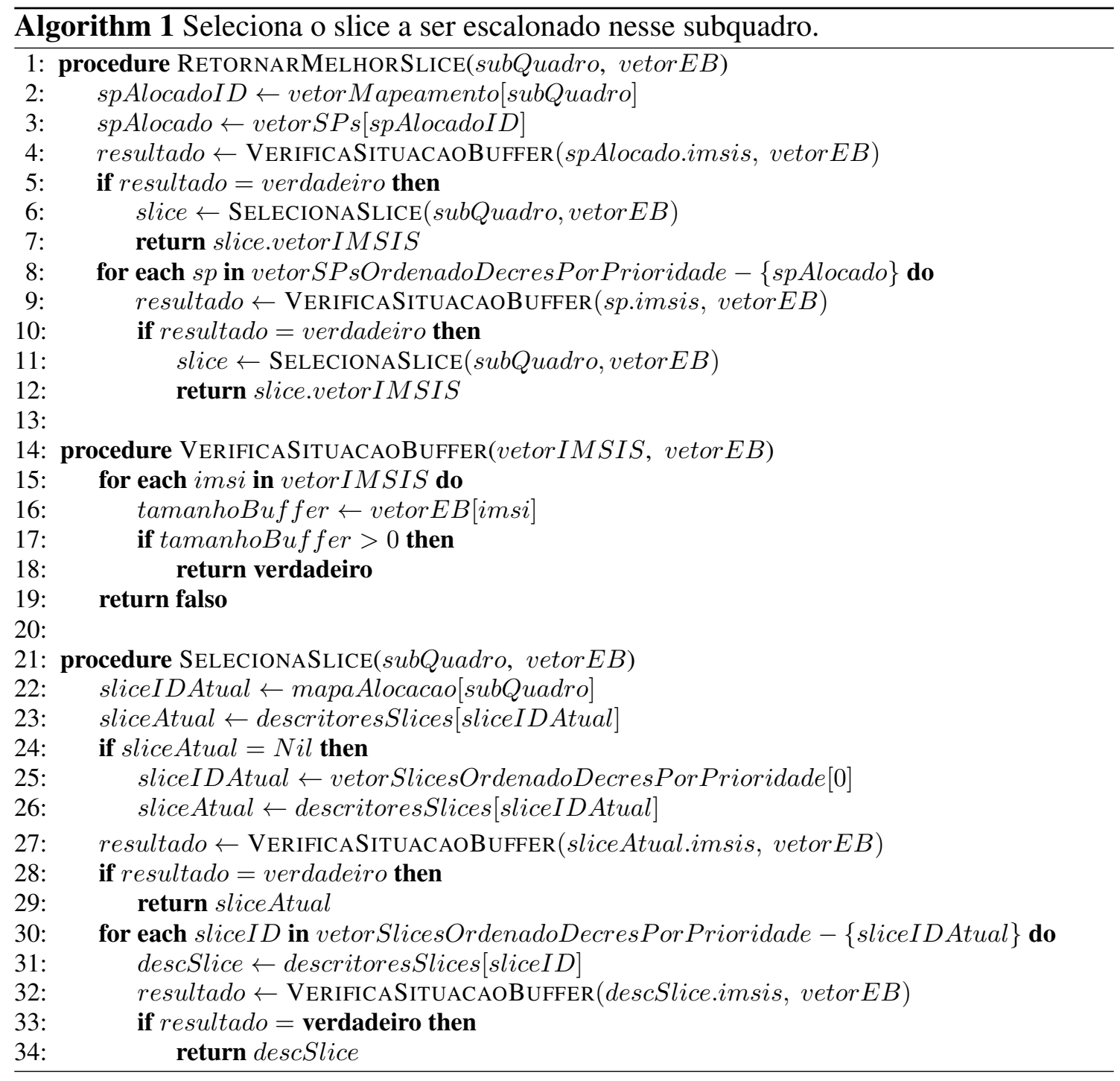

tenha dados a serem transmitidos.

\section{Avaliação de Desempenho}

Nesta seção é avaliado o desempenho de nossa proposta usando dois esquemas de network slicing, estático e dinâmico. No esquema estático, o OS, após escolher o SP a ser servido neste subquadro, a partir da alocação dos subquadros feita pelo PI, seleciona um slice desse SP a ser escalonado nesse subquadro. Mesmo que nenhum dos slices desse SP tenha dados a serem transmitidos neste subquadro, ainda assim um de seus slices precisa ser fornecido ao escalonador. No esquema dinâmico é dada prioridade de escalonamento a um dos slices pertencentes ao SP definido pelo PI neste subquadro. Entretanto, se nenhum dos slices desse SP possuir dados a serem transmitidos neste subquadro, o OS escolhe um SP de maior prioridade em que pelo menos um dos slices desse SP tenha tráfego a ser transmitido. Após escolher o SP, o OS seleciona o slice e envia-o ao escalonador. O esquema dinâmico é o definido no Algoritmo 1.

\subsection{Configuração do Cenário}

O cenário de simulação contém um eNB, dois SPs (veículos e evento), um EPC e um hospedeiro responsável por gerar tráfego de downlink na rede. Há diversos UEs na rede, 
variando de 20 a 50, com incrementos de 10. O framework Simulation of Urban MObility $(\mathrm{SUMO})^{3}$, versão 0.31 .0 , foi usado para gerenciar a mobilidade dos veículos. Foi empregado um trecho do cenário de Manhattan Grid, contendo 5 ruas de 2 pistas. Tais ruas são espaçadas por 100 metros, o que totaliza uma área de $160.000 \mathrm{~m}^{2}$.

O eNB está localizado no centro do cenário. Os UEs são uniformemente distribuídos no cenário. Um UE recebe apenas um tipo de tráfego. Os tipos de tráfego são: VoIP, FTP e Vídeo. Há 4 slices na rede, dois para cada SP. Os slices 1 e 2 pertencem ao SP responsável pelos veículos (SP-VEIC), enquanto que os slices 3 e 4 são do SP responsável pelo evento (SP-EVEN). Quatro subquadros de rádio foram alocados ao SP-VEIC, e seis subquadros ao SP-EVEN. Todos os participantes do evento andam a pé. Todo o tráfego tem como origem o hospedeiro, que está conectado ao EPC, e como destino os UEs. A largura de banda e o atraso da conexão cabeada entre o hospedeiro e o EPC são de 100Gb/s e $1 \mathrm{~ms}$, respectivamente. O tamanho do buffer RLC é de 150 KBytes, que é o recomendado para dispositivos da Categoria 1 [3GPP 2017]. Dispositivos da Categoria 1 suportam apenas SISO (Single Input, Single Output), que são aqueles usados na simulação. As Tabelas 1 e 2 sumarizam o modelo do tráfego e a descrição dos slices empregados na simulação.

Tabela 1. Modelo de Tráfego.

\begin{tabular}{|c|c|c|c|}
\hline Aplicação & VoIP & FTP & Vídeo \\
\hline Descrição & $\mathrm{G} .711^{1}$ & TCP Bulk ${ }^{2}$ & MPEG $4^{3}$ \\
\hline BitRate & $64 \mathrm{Kbps}$ & N/A & $742 \mathrm{Kbps}$ \\
\hline QCI & 1 & 9 & 4 \\
\hline \multicolumn{4}{|c|}{$\begin{array}{ll}1 & \text { Modelo On/Off. Tempo On }=0.352 ; \text { Tempo Off }=0.650 \\
2 & \text { Definido em: http: em:/www.nsnamorg/doxygen/tcp-bulk- } \\
\text { send_8cc_source.html. Foi empregado o TCP Vegas. } \\
3 \text { Baseado em trace. Nós usamoso trace ARD NEWS, que está dis- } \\
\text { ponível em: http://www2.tkn.tu-berlin.de/research/trace/ltvt.html. }\end{array}$} \\
\hline
\end{tabular}

Tabela 2. Descrição dos Slices.

\begin{tabular}{c||c|c|c|c}
\hline ID do Slice & 1 & 2 & 3 & 4 \\
\hline \hline Aplicação & VoIP & Vídeo & FTP & Vídeo \\
\hline Dono do Slice & VEÍC. & VEÍC. & EVENTO & EVENTO \\
\hline Subquadros & $\{3,5\}$ & $\{7,10\}$ & $\{1,6\}$ & $\{2,4,8,9\}$ \\
\hline Porcentagem de UEs & $75 \%$ & $10 \%$ & $5 \%$ & $10 \%$ \\
\hline
\end{tabular}

Foram empregados o modelo de propagação Kun e um trace do modelo de $f a$ ding para cenários suburbanos. O modelo de fading foi gerado pelo script fading-tracegenerator.m, que é disponibilizado pelo NS-3. A entidade HARQ está ativada. A Tabela 3 expõe os principais parâmetros usados na simulação.

\begin{tabular}{c||c}
\multicolumn{2}{c}{ Tabela 3. Parâmetros da Simulação. } \\
\hline Parâmetro & Valor \\
\hline Número de eNBs & 1 \\
\hline Área do Cenário & $160.000 \mathrm{~m}^{2}$ \\
\hline Frequência do Sistema & $2655 \mathrm{MHz}$ \\
\hline Modelo de Propagação & Kun $2600 \mathrm{Mhz}$ \\
\hline Potência de TX eNB & $34 \mathrm{dBm}$ \\
\hline Potência de TX UE & $10 \mathrm{dBm}$ \\
\hline Largura de Banda do Sistema & $10 \mathrm{MHz}$ \\
\hline Escalonador Downlink & Proportional Fair \\
\hline Modo RLC & Não Reconhecido (Unacknowledged Mode - UM) \\
\hline Tamanho do Buffer RLC & $150 \mathrm{KBytes}$ \\
\hline Velocidade dos UEs & $3 \mathrm{Km} / \mathrm{h}$ (A pé); $60 \mathrm{~km} / \mathrm{h}$ (veículo) \\
\hline Número de Replicações & 34 \\
\hline Duração da Simulação & $150 \mathrm{~s}$
\end{tabular}

\subsection{Resultados}

As figuras presentes nesta seção ilustram os valores médios por UE para as seguintes métricas de QoS: atraso, jitter, perdas e vazão. Todas essas métricas são expostas como função do número de UEs na rede. Foram realizados 8 experimentos, um para cada combinação de esquema de slicing e número de UEs na rede. Cada experimento foi executado 34 vezes com sementes distintas. Dessa forma, foram executadas 272 simulações. Nas figuras, as linhas contínuas representam o slicing dinâmico, enquanto que as linhas

\footnotetext{
${ }^{3}$ http://sumo.dlr.de/index.html
} 
tracejadas correspondem ao slicing estático. Os intervalos de confiança de $95 \%$ são mostrados nas figuras. Note que eles são bem pequenos e imperceptíveis nas figuras. Em todas as figuras, os Slices 1 e 2 pertencem ao SP-VEIC, enquanto que os Slices 3 e 4 são do SP-EVEN.

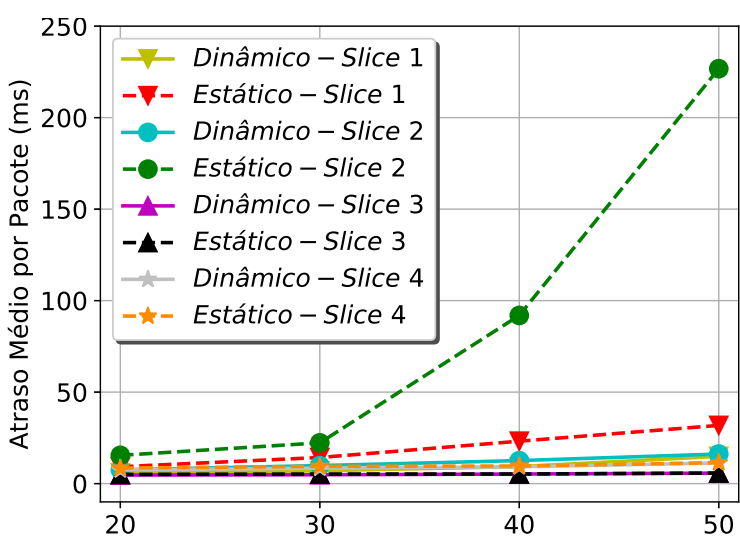

(a) Atraso Médio por Pacote.

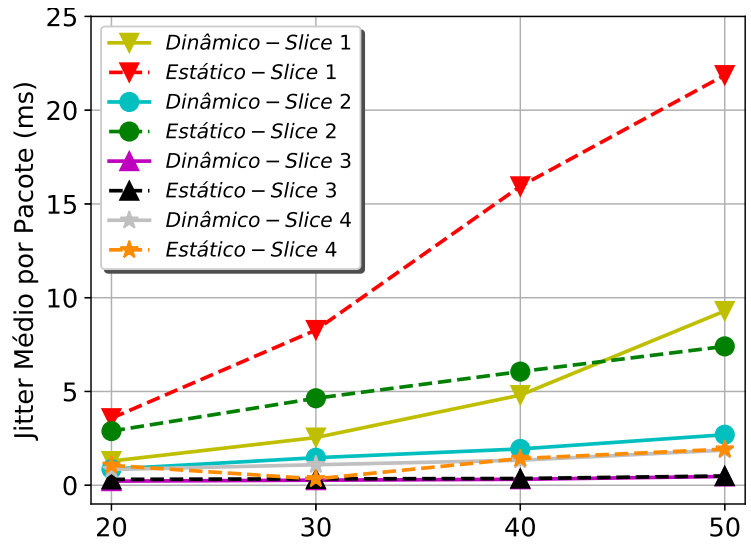

(b) Jitter Médio por Pacote .

Figura 3. Atraso e Jitter médio de cada pacote por UE em cada Slice como função do número de UEs na rede.

A Figura 3(a) apresenta o atraso médio de cada pacote por UE. Como pode ser observado na figura, tanto o slicing estático quanto o slicing dinâmico possuem resultados parecidos nos slices pertencentes ao SP-EVEN. Isso ocorre, pois os 6 subquadros alocados são suficientes para o tráfego do SP-EVEN, mesmo no cenário com 50 UEs na rede. Entretanto, os dispositivos dos slices pertencentes ao SP-VEIC têm o atraso de seus pacotes aumentado consideravelmente no slicing estático, em especial na rede com 50 UEs.

O slicing estático não permite que os subquadros alocados ao SP-EVEN possam ser usados pelos slices do SP-VEIC quando nenhum dos slices pertencentes ao SP-EVEN tenha dados a serem transmitidos. O slicing dinâmico, por outro lado, permite que o SP-VEIC use os recursos não usados pelo SP-EVEN, fazendo com que os pacotes do SP-VEIC sejam servidos pelo escalonador de modo mais rápido e, portanto, é reduzido consideravelmente o atraso percebido pelos UEs pertencentes ao SP-VEIC. Os UEs pertencentes ao Slice 2 são os que mais sofrem com o slicing estático, visto que seus pacotes carregam mais bytes em comparação com os pacotes do Slice 1, ocasionando um maior tempo de escalonamento. No cenário com 50 UEs, o atraso médio dos pacotes do Slice 2 é aproximadamente 16 vezes maior ( $226 \mathrm{~ms}$ contra $16 \mathrm{~ms}$ ) em comparação com os pacotes do Slice 2 no slicing dinâmico.

A Figura 3(b) expõe o jitter médio de cada pacote por UE. De modo similar à Figura 3(a), o slicing estático afeta negativamente os fluxos pertencentes ao SP-VEIC. Entretanto, diferentemente da Figura 3(a), o Slice 1 é o mais afetado pelo slicing estático. O Slice 1 também tem o pior desempenho em relação ao jitter dentre os 4 slices no slicing dinâmico. Esse maior jitter no Slice 1 deve-se a um conjunto de fatores. Primeiro, a maior prioridade de escalonamento do Slice 2 em relação ao Slice 1, o que faz com que no slicing dinâmico, o SP-VEIC priorize a alocação de recursos de subquadros não utilizados pelo SP-EVEN ao Slice 2.

Além disso, $75 \%$ dos UEs transmitem tráfego VoIP, o que faz com que em alguns 
subquadros de rádio muitos sejam os pacotes VoIP gerados ao mesmo tempo pelos fluxos VoIP, o que aumentaria o atraso de tais pacotes, visto o maior tempo para escaloná-los. Entretanto, em vários instantes poucos são os pacotes VoIP gerados ao mesmo tempo, acarretando um atraso menor a esses pacotes. Dessa forma, existem pacotes VoIP que são servidos rapidamente, enquanto alguns outros lentamente, o que, consequentemente, aumenta o jitter. No cenário com 50 UEs, o jitter médio no Slice 1 do slicing estático é cerca 2,3 vezes maior que o mesmo slice no slicing dinâmico (21 ms contra $9 \mathrm{~ms}$ ).

A Figura 4(a) ilustra a perda média de pacotes por UE. De modo similar às Figuras 3(a) e 3(b), os slices pertencentes ao SP-VEIC são afetados negativamente pelo slicing estático. De modo especial, o Slice 2 tem uma perda de pacotes bastante significativa quando há 50 UEs na rede. Conforme visto na Figura 3(a), no slicing estático, o atraso médio dos pacotes do Slice 2 aumenta consideravelmente à medida que cresce o número de UEs na rede. Com o aumento do atraso, mais pacotes vão sendo enfileirados nos buffers RLC do eNB, os quais são responsáveis por armazenar temporariamente os pacotes dos UEs. Os pacotes ficam nesses buffers até serem servidos pelo escalonador; ou, no pior caso, até serem descartados.

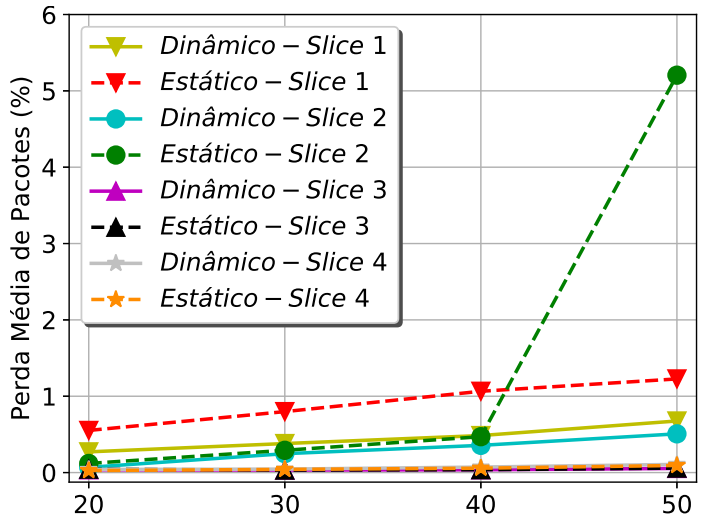

(a) Perda Média de Pacotes.

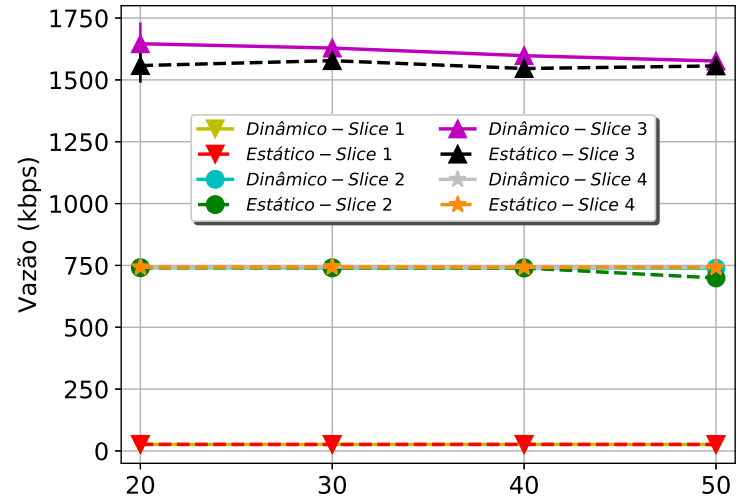

(b) Vazão Média.

Figura 4. Perdas de Pacotes e Vazão por UE em cada Slice como função do número de UEs na rede.

Como os pacotes do Slice 2, no slicing estático, tem um alto atraso e, além disso, o tamanho dos pacotes de vídeos carregam muitos bytes, os buffers RLC ficam cheios. Quando esses buffers enchem, pacotes precisam ser descartados, conforme mostrado na Figura 4(a). Por outro lado, no slicing dinâmico, as perdas de pacotes são relativamente baixas. O Slice 2 no cenário com 50 UEs, por exemplo, tem no slicing estático uma perda de aproximadamente $5,2 \%$ de pacotes, enquanto que no slicing dinâmico apenas $0,5 \%$ dos pacotes são descartados, uma quantidade dez vezes menor.

A Figura 4(b) apresenta a vazão média dos UEs. De modo geral, a vazão média dos UEs em cada slice, independente do esquema de slicing usado, é basicamente a mesma. Embora aparentemente o slicing dinâmico ofereça uma vazão superior, de modo especial no Slice 3, essa vantagem do slicing dinâmico sobre o slicing estático não é estatisticamente significativa. Entretanto, quando há 50 UEs no cenário, a vazão média dos UEs no Slice 2 usando o slicing dinâmico é ligeiramente superior que no slicing estático. Isso ocorre, pois como visto na Figura 4(a), o slicing estático aumenta consideravelmente a quantidade de pacotes perdidos no Slice 2 em comparação com o slicing dinâmico. Dessa forma, a vazão dos UEs no Slice 2 precisa ser necessariamente menor no slicing 
estático, o que realmente acontece, conforme exposto na Figura 4(b). Vale mencionar que no Slice 1, em ambos os esquema de slicing, a vazão é de no máximo $23 \mathrm{Kbps}$, uma vez que a aplicação VoIP só transmite dados quando no estado ON.

A partir dos resultados obtidos neste trabalho, pode-se concluir que o esquema de slicing dinâmico é superior ao slicing estático. Tendo em vista que o estado de uma rede de comunicação de dados altera-se com frequência, bem como nem sempre os operadores de rede aplicam uma política de alocação de recursos correta, o Otimizador de Slices, ao utilizar o slicing dinâmico, adapta os slices ao estado da rede, permitindo que recursos de rádio não usados por SPs sejam alocados a outros SPs que necessitam de tais recursos e, consequentemente, há uma melhora na QoS ofertada ao usuário final.

\section{Conclusões e Trabalhos Futuros}

Neste artigo foi apresentado o Otimizador de Slices, um componente que realiza o conceito de network slicing no canal downlink das redes LTE para múltiplos Provedores de Serviço. Os Provedores de Serviço constroem seus slices e definem o escalonamento deles por meio de um módulo de orquestração próprio, que reside em um controlador vSDN. A partir de informações recebidas sobre slices do controlador vSDN e do estado da rede, 0 Otimizador de Slices seleciona o melhor slice a ser escalonado no momento. Simulações foram realizadas para mostrar que o Otimizador de Slices usa eficientemente os recursos de rádio downlink e, consequentemente, melhora a QoS percebida pelos usuários finais.

Como trabalhos futuros, nós planejamos permitir ao Otimizador de Slices manipular slices de múltiplos SPs no canal de rádio uplink. Além do mais, esperamos integrar funções virtualizadas de rede nos slices e avaliar o overhead causado pela comunicação entre os componentes de nossa arquitetura.

\section{Agradecimentos}

Os autores gostariam de agradecer a CAPES pela bolsa de doutorado. Este trabalho é parte do INCT sobre Internet do Futuro para Cidades Inteligentes (CNPq 465446/2014-0, CAPES 88887.136422/2017-00 e FAPESP 2014/50937-1).

\section{Referências}

3GPP (2017). Disponível em: http://http://www.etsi.org/deliver/etsi_ts/136300_136399/136306/10.02.00_60/.

Al-Shabibi, A., De Leenheer, M., Gerola, M., Koshibe, A., Parulkar, G., Salvadori, E., and Snow, B. (2014). OpenVirteX: Make Your Virtual SDNs Programmable. In Proceedings of the Third Workshop on Hot Topics in Software Defined Networking, HotSDN '14, pages 25-30, New York, NY, USA. ACM.

Blenk, A., Basta, A., Reisslein, M., and Kellerer, W. (2016). Survey on Network Virtualization Hypervisors for Software Defined Networking. IEEE Communications Surveys Tutorials, 18(1):655-685.

Caballero, P., Banchs, A., de Veciana, G., and Costa-Pérez, X. (2017). Network slicing games: Enabling customization in multi-tenant networks. In IEEE INFOCOM 2017 IEEE Conference on Computer Communications, pages 1-9.

Chartsias, P. K., Amiras, A., Plevrakis, I., Samaras, I., Katsaros, K., Kritharidis, D., Trouva, E., Angelopoulos, I., Kourtis, A., Siddiqui, M. S., Viñes, A., and Escalona, 
E. (2017). SDN/NFV-based end to end network slicing for 5G multi-tenant networks. In 2017 European Conference on Networks and Communications (EuCNC), pages 1-5.

Choyi, V. K., Abdel-Hamid, A., Shah, Y., Ferdi, S., and Brusilovsky, A. (2016). Network slice selection, assignment and routing within 5G Networks. In 2016 IEEE Conference on Standards for Communications and Networking (CSCN), pages 1-7.

Foukas, X., Patounas, G., Elmokashfi, A., and Marina, M. K. (2017). Network Slicing in 5G: Survey and Challenges. IEEE Communications Magazine, 55(5):94-100.

Grøndalen, O., Zanella, A., Mahmood, K., Carpin, M., Rasool, J., and Østerbø, O. N. (2017). Scheduling Policies in Time and Frequency Domains for LTE Downlink Channel: A Performance Comparison. IEEE Transactions on Vehicular Technology, 66(4):3345-3360.

Haque, I. T. and Abu-Ghazaleh, N. (2016). Wireless Software Defined Networking: A Survey and Taxonomy. IEEE Communications Surveys Tutorials, 18(4):2713-2737.

Hu, M., Chang, Y., Sun, Y., and Li, H. (2016). Dynamic slicing and scheduling for wireless network virtualization in downlink LTE system. In 2016 19th International Symposium on Wireless Personal Multimedia Communications (WPMC), pages 153158.

Kamel, M. I., Le, L. B., and Girard, A. (2014). LTE Wireless Network Virtualization: Dynamic Slicing via Flexible Scheduling. In 2014 IEEE 80th Vehicular Technology Conference (VTC2014-Fall), pages 1-5.

Kokku, R., Mahindra, R., Zhang, H., and Rangarajan, S. (2013). CellSlice: Cellular wireless resource slicing for active RAN sharing. In 2013 Fifth International Conference on Communication Systems and Networks (COMSNETS), pages 1-10.

Kreutz, D., Ramos, F. M. V., Veríssimo, P. E., Rothenberg, C. E., Azodolmolky, S., and Uhlig, S. (2015). Software-Defined Networking: A Comprehensive Survey. Proceedings of the IEEE, 103(1):14-76.

LENA (2017). Disponível em: http://networks.cttc.es/mobile-networks/softwaretools/lena/.

McKeown, N., Anderson, T., Balakrishnan, H., Parulkar, G., Peterson, L., Rexford, J., Shenker, S., and Turner, J. (2008). OpenFlow: Enabling Innovation in Campus Networks. SIGCOMM Comput. Commun. Rev., 38(2):69-74.

Mijumbi, R., Serrat, J., Gorricho, J. L., Bouten, N., Turck, F. D., and Boutaba, R. (2016). Network Function Virtualization: State-of-the-Art and Research Challenges. IEEE Communications Surveys Tutorials, 18(1):236-262.

NS-3 (2017). Disponível em: https://www.nsnam.org.

Parsaeefard, S., Jumba, V., Derakhshani, M., and Le-Ngoc, T. (2015). Joint resource provisioning and admission control in wireless virtualized networks. In 2015 IEEE Wireless Communications and Networking Conference (WCNC), pages 2020-2025.

Rezende, P. and Madeira, E. (2018). An adaptive network slicing for LTE Radio Access Networks. In 2018 Wireless Days.

Samdanis, K., Costa-Perez, X., and Sciancalepore, V. (2016). From network sharing to multi-tenancy: The 5G network slice broker. IEEE Communications Magazine, 54(7):32-39. 